\title{
Intraluminal aortic manipulation by means of intra-aortic filter, cannulation, and external clamp maneuvers evaluated versus dislodged embolic material
}

\author{
Patrik Boivie, MD, ${ }^{a}$ Magnus Hansson, MD, PhD, ${ }^{b}$ and Karl Gunnar Engström, MD, PhD, FRCS ${ }^{a}$
}

From the Department of Surgical and Perioperative Science, Heart Center, Cardiothoracic Division, ${ }^{a}$ and the Department of Medical Biosciences, Section for Pathology, ${ }^{\mathrm{b}}$ Umeå University Hospital, Umeå, Sweden.

There were no commercial sources of funding. In scientific terms, the work was supported by the Swedish Society for Medical Research; Funds of the Medical Faculty, Umeå University Hospital; the Heart Foundation of Northern Sweden; and the Swedish Stroke Foundation.

Received for publication Sept 1, 2005; revisions received Sept 30, 2005; accepted for publication Oct 3, 2005

Address for reprints: Patrik Boivie, MD, Department of Surgical and Perioperative Science, Heart Center, Cardiothoracic Division, Umeå University Hospital, S-901 85 Umeå, Sweden (E-mail: patrik.boivie@ vll.se).

J Thorac Cardiovasc Surg 2006;131:283-9

$0022-5223 / \$ 32.00$

Copyright () 2006 by The American Association for Thoracic Surgery

doi:10.1016/j.jtcvs.2005.10.015
Objectives: Aortic atherosclerosis is an important risk factor for cerebrovascular accidents in cardiac surgery. An intra-aortic filter might reduce this risk. We aimed to analyze the risks for emboli associated with intraluminal aortic manipulation and intra-aortic filter handling in relation to cannulation and external clamp maneuvers.

Methods: A model was designed with a cadaver aorta and retrograde perfusion $(\mathrm{n}=$ 16). A crossclamp was positioned on the ascending aorta and repeatedly opened under pressure to collect aliquots with dislodged particles. Cannulation was performed after 10 clamp maneuvers, followed by positioning and removing the intra-aortic filter, with each step followed by a washout sequence to collect perfusate. The removed filter was also analyzed. Evaluation was by means of digital image analysis, with differentiation of particles into different spectra.

Results: Intra-aortic filter manipulation produced a significant washout of embolic particles; in particular, this was seen for the macroscopic cellular spectrum $(P=$ .006 and $P=.002$ for filter insertion and removal, respectively). Particles were also found to be collected by the filter $(P=.004)$. In addition, cannulation and aortic crossclamp manipulation generated a notable number of particles $(P=.001$ and $P=.013$, respectively).

Conclusions: The intra-aortic filter collects material during aortic manipulation. However, intraluminal aortic manipulation from filter handling can also dislodge particles, possibly related to shedding of intimal debris. This is in addition to substantial amounts of particles that are generated by aortic cannulation and aortic crossclamping.

I $\mathrm{n}$ the general population, atherosclerosis of the aorta is associated with cerebrovascular accidents (CVAs), ${ }^{1}$ being a well-known risk in cardiac surgery. The issue also has relevance for percutaneous coronary intervention (PCI) from a perspective of intraluminal aortic manipulation. ${ }^{2}$ The link between catheter intervention and dislodged particles has been confirmed by means of transcranial ultrasonography. ${ }^{3}$ For cardiac surgery, risks for embolic phenomena with external aortic clamp manipulation are well established ${ }^{4-7}$ and confirmed by means of ultrasonography. ${ }^{8}$

Several new methods have been developed to try to improve outcomes in patients undergoing cardiac surgery with reference to CVAs. An intra-aortic filter (IAF) has been demonstrated to capture and remove embolic material and thereby protect the brain during aortic manipulation. ${ }^{9-11}$ The pattern of embolic material produced by aortic crossclamping (ACC) has been described in a perfusion model of the cadaveric aorta. ${ }^{12}$ The present study uses the same perfusion model with the aim to evaluate risks associated with intraluminal aortic manipulation by the IAF and its 

Abbreviations and Acronyms
$\mathrm{ACC}=$ aortic crossclamping
$\mathrm{CVA}=$ cerebrovascular accident
$\mathrm{IAF}=$ intra-aortic filter
PCI = percutaneous coronary intervention

possible contribution to particle embolization. The effects of cannulation and clamp maneuvers were studied in parallel.

\section{Methods \\ Subjects}

Seventeen autopsy subjects selected from a high-risk group with atherosclerosis were included in the study (Table 1). None of the subjects had been exposed to cardiac surgery, whereas one had a history of PCI before death. In one experiment on a subject with severe atherosclerosis, the aortic wall ruptured at cannulation. This subject was rejected, leaving a total of 16 observations. The study was approved by the Umeå University ethical committee (Dnr 01-142).

\section{Aortic Perfusion Model}

We developed an aortic perfusion model using cadaveric aorta, as previously described. ${ }^{12}$ In this study the model was further developed and modified to evaluate intimal manipulation caused by IAF, as well as aortic cannulation. A schematic view of the experimental model is shown in Figure 1. In brief, retrograde washout aliquots were collected from the open end of the ascending aorta downstream from the IAF, cannulation site, and ACC. The aorta was removed from the body and dissected free from surrounding tissue, side branches were ligated, and a venous-type cannula (32F, Polystan A/S) was introduced through the descending part of the aorta. Perfusion medium $(9 \mathrm{~g} / \mathrm{L} \mathrm{NaCl})$ was allowed to flush the inside of the aorta before further experimentation, of which the last fraction was sampled to represent baseline. A standard 70-mm aortic crossclamp (Pilling Co) was applied to the ascending aorta and locked in position to the aortic adventitia with multiple stitches. The aorta was pressurized at $60 \mathrm{~mm} \mathrm{Hg}$ from the hydrostatically elevated perfusion medium reservoir. The diameter of the pressurized aorta was measured to guide the selection of IAF size. A baseline sample was collected before any manipulation, followed by 10 repeated ACC maneuvers to collect washout aliquots of about $50 \mathrm{~mL}$ each. Purse-string sutures were applied as in routine cardiac surgery, followed by cannulation (Embol-X cannula) and a repeated ACC release to collect a washout sample. The IAF (Embol-X filter) was inserted into the pressurized aorta, according to routine surgical procedures and manufacturer's recommendations and with an additional ACC release and sample, followed by IAF removal and a final ACC washout sample. The removed filter was everted and stirred in a test tube with $50 \mathrm{~mL}$ of saline to collect any captured particles.

\section{Processing of Washout Samples}

The samples were processed essentially as previously described. ${ }^{12}$ Each aliquot was centrifuged, the supernatant was carefully aspi-
TABLE 1. Subject demographics, autopsy data, aortic characteristics, and cause of death

\begin{tabular}{|c|c|c|c|}
\hline & n (\%) & Mean \pm SEM & Range \\
\hline Age (y) & & $69.8 \pm 2.88$ & $48-87$ \\
\hline Male sex & $13(81)$ & & \\
\hline \multicolumn{4}{|l|}{ Clinical diagnosis } \\
\hline History of CVA & $4(25)$ & & \\
\hline Diabetes mellitus & $2(13)$ & & \\
\hline Hypertension & $4(25)$ & & \\
\hline \multicolumn{4}{|l|}{ Cardiac data at autopsy } \\
\hline Coronary disease & $10(63)$ & & \\
\hline Valvular disease & $7(44)$ & & \\
\hline $\begin{array}{l}\text { Previous myocardial } \\
\text { infarction }\end{array}$ & $5(31)$ & & \\
\hline \multicolumn{4}{|l|}{$\begin{array}{l}\text { Aortic observations at } \\
\text { autopsy }\end{array}$} \\
\hline Aortic diameter (mm) & & $32.1 \pm 0.64$ & $28.0-38.0$ \\
\hline $\begin{array}{l}\text { Aortic media wall } \\
\text { thickness (mm) }\end{array}$ & & $1.7 \pm 0.08$ & 1.3-2.3 \\
\hline $\begin{array}{l}\text { Magnitude of } \\
\text { calcification (0-4) }\end{array}$ & & $1.9 \pm 0.18$ & $1-3$ \\
\hline $\begin{array}{l}\text { Ulcerative } \\
\text { calcifications }\end{array}$ & $8(50)$ & & \\
\hline \multicolumn{4}{|l|}{ Cause of death } \\
\hline $\begin{array}{l}\text { Acute myocardial } \\
\text { infarction }\end{array}$ & $5(31)$ & & \\
\hline $\begin{array}{l}\text { Circulatory failure, } \\
\text { unspecific }\end{array}$ & $3(19)$ & & \\
\hline $\begin{array}{l}\text { Non-cardiac-related } \\
\text { death }\end{array}$ & $8(50)$ & & \\
\hline
\end{tabular}

SEM, standard error of the mean; CVA, cerebrovascular accident.

rated, and the particle deposit was resuspended in $50 \mathrm{~mL}$ of deionized-filtered water to lyse remaining erythrocytes. After a repeated centrifugation, the deposit was fixed for 10 minutes at $22^{\circ} \mathrm{C}$ by means of resuspension in $4 \%$ formaldehyde in phosphate buffer. Fixation was stopped by means of an additional recentrifugation and suspension in water. The above steps were done in conjunction with the perfusion experiment. After centrifugation and aspiration of supernatant, the deposit was stained with Giemsa ( $20 \mu \mathrm{L}$ for 10 minutes at $22^{\circ} \mathrm{C}$ ), followed by 2 additional washingcentrifugation cycles. The deposit was spread out on a microscopic slide and left to dry at room temperature. In case of extensive particle load, additional slides were used to avoid severely condensed material that would hamper image analysis.

\section{Image Analysis of Particles}

Particles were viewed both macroscopically and microscopically, essentially as previously described, ${ }^{12}$ to which reference is made for further details. The macroscopic view encloses large particles that would not be identified under the microscope and vice versa. Furthermore, there was differentiation between dense particles (eg, opaque and nontransparent spectrum of gray scale), which were referred to as calcified particles, and transparent spectrum, which were referred to as cellular particles. 


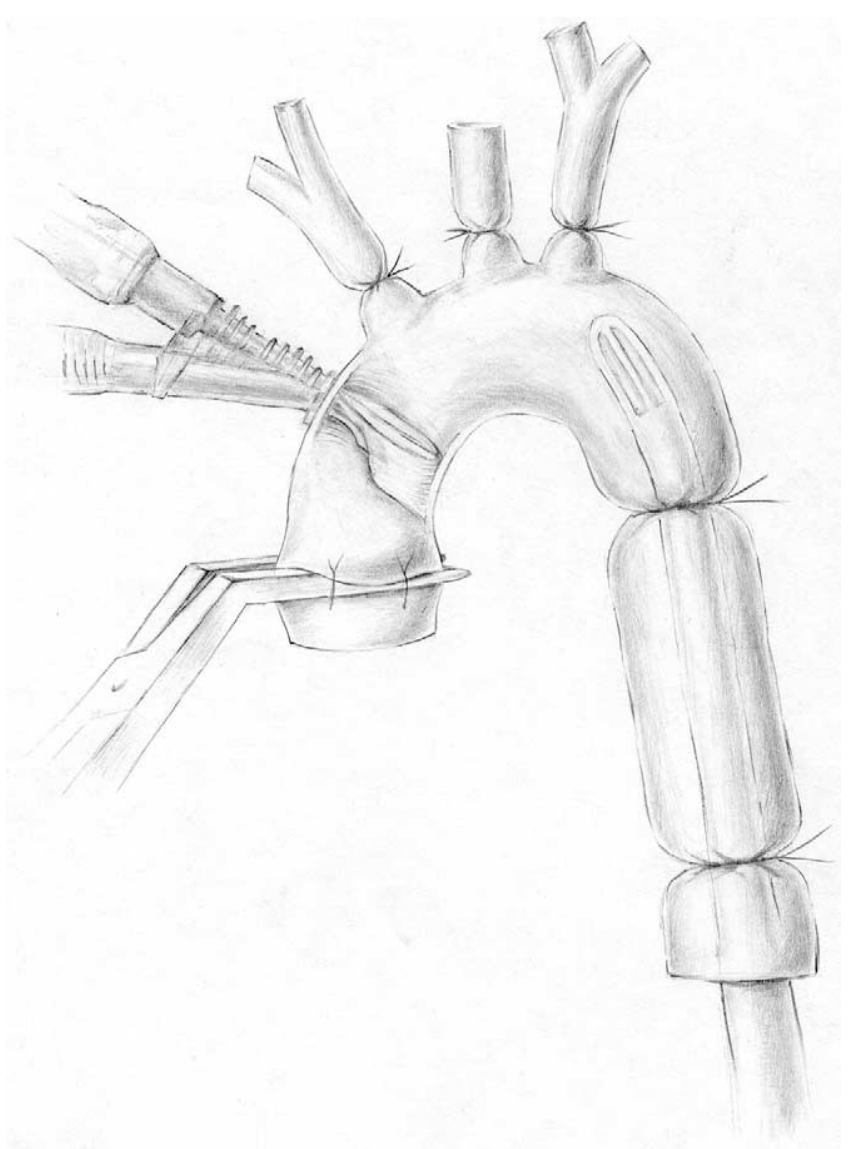

Figure 1. Schematic view of the aortic preparation and experimental model. See the Methods section for details. (IIlustration by Ankin Güvencel).

We have introduced several methodological improvements in image analysis of particles since our previous report using this cadaveric model. ${ }^{12}$ The cellular spectrum here did not overlap the dense calcified spectrum in threshold settings of the image analyzer. The new routine allowed an easier comparison between groups. Another difference is the subdivision into cellular and calcified particles for both the macroscopic and microscopic appearance, rather than for microscopic evaluation only. The programming of the image analyzer included a background subtraction to compen- sate for skewed illumination but was in other terms in accordance with our previous technique. ${ }^{12}$ The macroscopic digital evaluation overviewed the entire sample of particles and was merged with data from other slides if more than one glass slide was used. For the microscopic evaluation, triplicate views per slide were selected at random. The observer was blinded to the identity of the sample.

\section{Processing of Particle Data}

The scientific aim here focuses on IAF and intimal aortic manipulation. However, the design of the experiment required an ACC to pressurize the aorta and make it clinically relevant. For this reason, the experiment was initiated by 10 repeated ACC maneuvers to dislodge and wash the clamp site free from particles that would contaminate the following steps of experimentation. The cannulation and IAF maneuvers were superimposed onto those particles caused by the simultaneous use of ACC. The initial ACC maneuvers and the decrease in particle output were used to predict the background interference during cannulation and IAF by calculating a linear regression curve, with a slope value provided in Table 2 .

Cannulation and IAF-specific particle output was possible to calculate from the difference against the ACC background slope, being extrapolated and subtracted from the cannulation and IAF manipulation. The difference is referred to as d-particles.

\section{Aortic Characteristics}

After perfusion, each aortic specimen was removed and cut open along its major curvature to describe characteristics of the aortas. The degree of atherosclerotic lesions was semiquantitatively graded $(0=$ normal to $4=$ porcelain aorta), as previously described. ${ }^{12}$ The presence of ulcerative plaques, aortic diameter, and wall thickness were measured.

\section{Statistical Analysis}

Because of a right-skewed distribution of particle counts and variability between subjects, raw data were logarithmically transformed, after which the paired 2-tailed Student $t$ test was applied throughout. A few samples of macroscopic analysis contained no visible particle debris. In the logarithmic transformation these were replaced by the nearest zero value of 0.1 that logarithmically becomes -1 . The next higher value in the data set was 1 , which results in a 0 value after transformation. The routine generated a close to normal distribution after logarithmic correction. Statistica (StatSoft) version 6.1 was used throughout.

TABLE 2. Particle output at aortic crossclamp opening (log)

\begin{tabular}{|c|c|c|c|c|c|c|}
\hline & $\begin{array}{c}\text { Baseline } \\
\text { particles (n) }\end{array}$ & $\begin{array}{c}\text { ACC } 1 \\
\text { particles (n) }\end{array}$ & $\begin{array}{c}P \\
\text { value }\end{array}$ & ACC 1-10 slope & $\begin{array}{c}P \\
\text { value }\end{array}$ & $\begin{array}{c}\text { Diameter } \\
(\mathrm{mm})\end{array}$ \\
\hline Macroscopic calcified & $0.23 \pm 0.24$ & $0.71 \pm 0.15$ & .060 & $-0.040 \pm 0.017$ & .029 & 6.12 \\
\hline Macroscopic cellular & $0.99 \pm 0.23$ & $1.59 \pm 0.19$ & .013 & $-0.076 \pm 0.021$ & .002 & 16.10 \\
\hline Microscopic calcified & $0.33 \pm 0.19$ & $0.77 \pm 0.17$ & .014 & $-0.029 \pm 0.012$ & .025 & 0.68 \\
\hline Microscopic cellular & $0.35 \pm 0.15$ & $0.65 \pm 0.11$ & .023 & $-0.037 \pm 0.011$ & .004 & 0.69 \\
\hline
\end{tabular}

Data are expressed on the logarithmic scale as mean values \pm standard error of the mean. $P$ values for ACC 1 refer to difference versus baseline. ACC, aortic cross clamp (numerals refer to ACC release sequence). Slope denotes a linear regression of particle output versus consecutive release of ACC (1-10) and difference to zero. Diameter values refer to maximum-size recordings in individual subjects during ACC manipulation. 
a) Macroscopic calcified particles

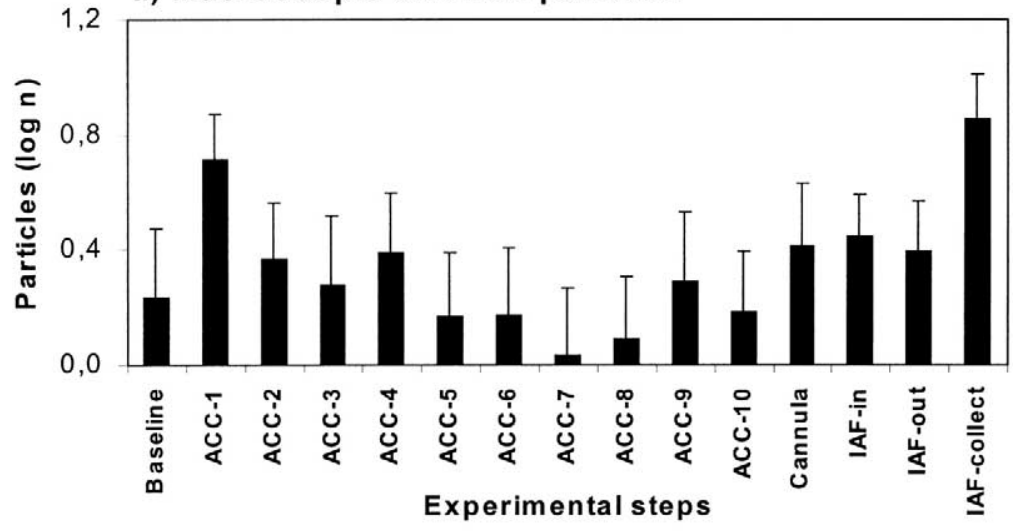

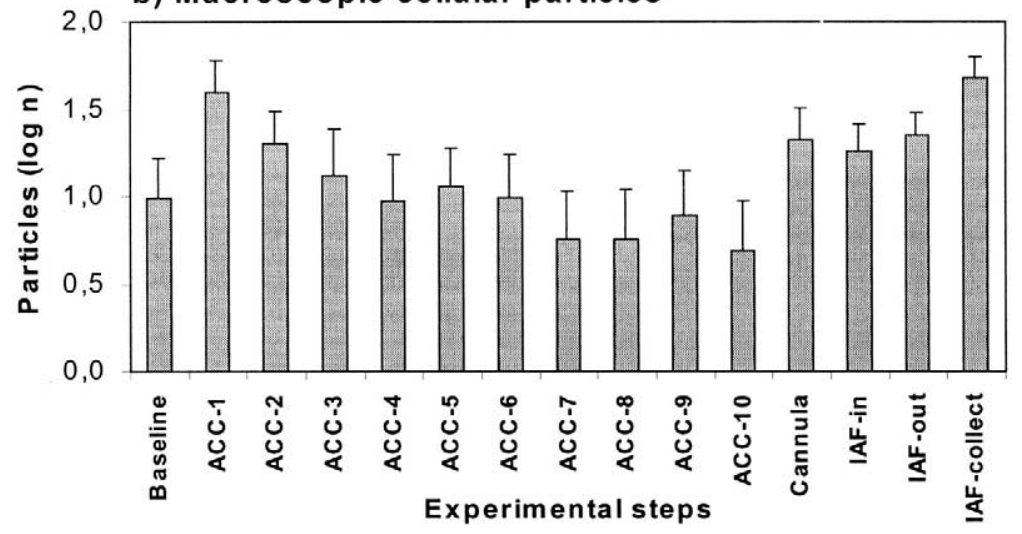

Figure 2. Logarithmic number of macroscopic particles produced by aortic manipulation. See Tables 2 and 3 for statistics and the Methods section for description. A, Macroscopic calcified particles; B, macroscopic cellular particles. ACC, Aortic crossclamp; IAF, intra-aortic filter.

\section{Results}

\section{Aortic Appearance}

All subjects had visible aortic calcification on the postmortem examination. Half of the aortic specimens had ulcerative plaque characteristics, whereas the remaining subjects had plaques of a more solid nature (Table 1). The majority of subjects were men, coronary and valvular disease were common, and with many concomitant risk factors of previous CVA, diabetes mellitus and hypertension. These characteristics are shared with routine cardiac-surgery patients.

\section{ACC Manipulation}

The initial ACC maneuver gave rise to a significant output of particles above baseline levels. This phenomenon was apparent for all spectra of analysis $(P=.013$ to .023$)$ except macroscopic calcified particles $(P=.060$, Table 2$)$. With repeated ACC, the number of particles significantly decreased, as demonstrated by a negative slope value of particles versus steps of ACC that was significant for all tested spectra (Table 2 and Figures 2 and 3). The maximum particle diameter extracted from all ACC procedures is presented in Table 2.

\section{Cannulation and IAF Manipulation}

The different spectra of particle analysis (macroscopicmicroscopic and calcified-cellular) showed a consistent pattern in relation to manipulation (Figures 2 and 3). Cannulation caused a significant increase in particles above the ACC background, regardless of spectral subdivision (Table $3)$. Intraluminal manipulation by introducing the IAF resulted in a significant output of particles $(P=.006$ to .043$)$ except for microscopic calcified debris (Table 3 ). The same pattern was observed at IAF removal with reference to macroscopic and microscopic cellular particles (Table 3). Particles collected in the IAF were measured. In all experiments the IAF contained particles, with the exception of one subject in the spectra of macroscopic calcified debris. The IAF collected significantly $(P=.004)$ more macroscopic cellular particles than were washed out when the filter was in position. A similar pattern but with borderline significance $(P=.050)$ was observed for microscopic calcified debris (Table 3 ).

The maximum diameter size of particles regardless of manipulation type is found in Table 3. With reference to IAF insertion and removal only, the maximum-size particles 
a) Microscopic calcified particles

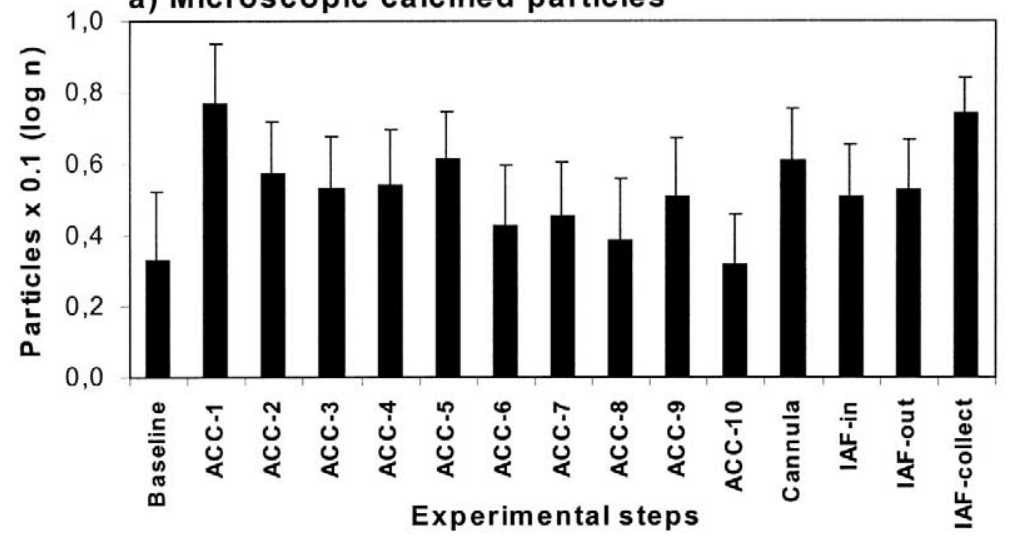

Figure 3. Logarithmic number of microscopic particles produced by aortic manipulation. See Tables 2 and 3 for statistics and the Methods section for description. A, Microscopic calcified particles $(x$ $0.1) ; B$, microscopic cellular particles $(\times 0.01)$. ACC, Aortic crossclamp; IAF, intra-aortic filter. b) Microscopic cellular particles

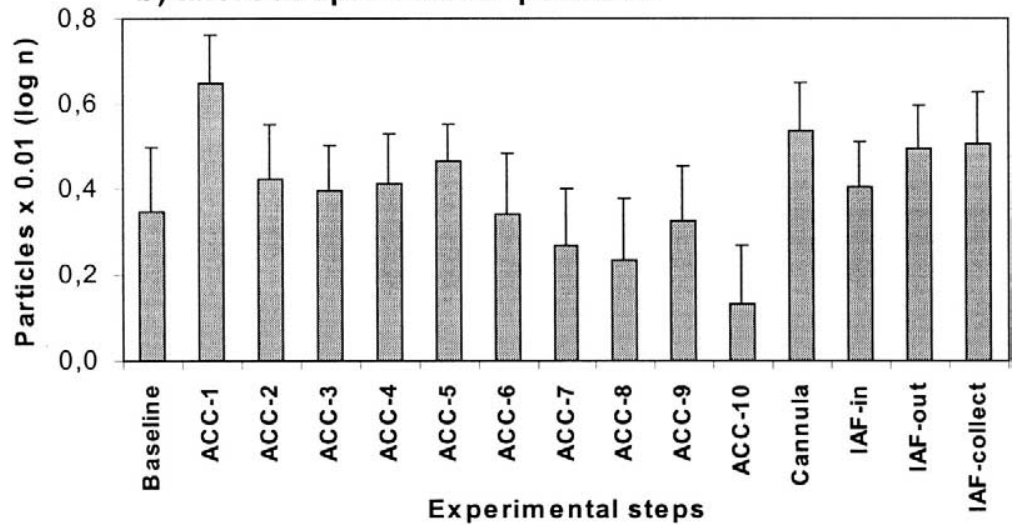

were as follows: $3.20 \mathrm{~mm}, 9.26 \mathrm{~mm}, 0.42 \mathrm{~mm}$, and 0.49 $\mathrm{mm}$, respectively, listed in the same order as in Table 3.

\section{Discussion}

Aortic atherosclerosis is a well-known predictor of CVA. ${ }^{4,5,13,14}$ With increasing age of patients undergoing cardiac interventions with operations or PCI, aortic calcification is a growing risk factor. Different technical solutions have been developed to prevent aortic emboli-related CVAs, such as ultrasonographic scanning to visualize a degenerated aorta during surgical intervention. Another approach is to capture potential particulate emboli by using a screen filter in the aorta (eg, IAF). Although this intervention is known to capture particles, ${ }^{9-11}$ the IAF introduces a new type of intraluminal aortic manipulation. The present study was aimed to further elucidate possible hazards with aortic cannulation, as well as ACC.

In a prospectively controlled study, the IAF was shown to decrease the total incidence of general neurologic adverse events, ${ }^{15}$ whereas CVAs were not significantly reduced,

TABLE 3. Manipulation-specific particle output (log)

\begin{tabular}{|c|c|c|c|c|c|c|c|c|c|}
\hline & $\begin{array}{l}\text { Cannulation } \\
\text { d-particles (n) }\end{array}$ & $\begin{array}{c}P \\
\text { value }\end{array}$ & $\begin{array}{c}\text { IAF in } \\
\text { d-particles (n) }\end{array}$ & $\begin{array}{c}P \\
\text { value }\end{array}$ & $\begin{array}{c}\text { IAF out } \\
\text { d-particles (n) }\end{array}$ & $\begin{array}{c}P \\
\text { value }\end{array}$ & $\begin{array}{c}\text { IAF collect } \\
\text { d-particles (n) }\end{array}$ & $\begin{array}{c}P \\
\text { value }\end{array}$ & $\begin{array}{c}\text { Diameter } \\
(\mathrm{mm})\end{array}$ \\
\hline Macroscopic calcified & $0.36 \pm 0.14$ & .023 & $0.43 \pm 0.19$ & .043 & $0.42 \pm 0.21$ & .063 & $0.41 \pm 0.20$ & .054 & 3.95 \\
\hline Macroscopic cellular & $0.72 \pm 0.18$ & .001 & $0.73 \pm 0.23$ & .006 & $0.90 \pm 0.23$ & .002 & $0.42 \pm 0.12$ & .004 & 9.26 \\
\hline Macroscopic calcified & $0.25 \pm 0.11$ & .038 & $0.18 \pm 0.11$ & .142 & $0.22 \pm 0.14$ & .136 & $0.23 \pm 0.11$ & .050 & 0.55 \\
\hline Microscopic cellular & $0.37 \pm 0.11$ & .004 & $0.27 \pm 0.09$ & .011 & $0.40 \pm 0.14$ & .014 & $0.10 \pm 0.08$ & .219 & 0.59 \\
\hline
\end{tabular}

Data are expressed on the logarithmic scale as mean values \pm standard error of the mean. The term d-Particles for cannulation, IAF in, and IAF out refers to the difference in recorded particles versus the ACC background slope value of Table 2 (see Methods section for further details). IAF collect denotes the difference between particles collected in the extracted filter versus washed-out particles when the filter was in position (IAF-In). Diameter values refer to maximum-size recordings in individual subjects and for the listed types of manipulation. 
possibly because of a limited statistical power. ${ }^{15}$ In a randomized study it was concluded that the IAF was safe and successful in collecting embolic debris and in preventing renal failure in a subgroup of high-risk patients, although the study was unable to identify a reduction in CVAs. ${ }^{16} \mathrm{~A}$ phenomenon that might explain unsuccessful CVA outcome is the possible role of aortic intimal manipulation caused by the IAF, which we have examined in this study. In a study by Banbury and colleagues, ${ }^{16}$ epiaortic scanning was performed to investigate intimal abrasion caused by the IAF. In $6.5 \%$ of the patients with IAFs, endothelial disruption was observed compared with $1.4 \%$ in the control group $(P<.001){ }^{16}$

In our perfusion model the IAF handling generated both macroscopic and microscopic particles. Particles were further subdivided on the basis of their transparency into calcified versus cellular particles. IAF insertion caused a washout of all types of debris, except for microscopic calcified particles. This phenomenon suggests that particles were dislodged at the circumference of the IAF and escaped filter capture. The IAF indeed captured particles in our model. However, the experiment was not designed to answer this question in view of a retrograde perfusion that differed from IAF use during intraoperative conditions. IAF-captured particles were likely to have been dislodged during IAF insertion. The aortic arch had been flushed before the experimentation. Furthermore, background particles were measured and subtracted to yield the IAF-specific particle count. This was made possible by the experimental steps before the IAF maneuvers, which were used to calculate a background regression curve. The removal of the IAF was associated with an additional washout of particles. In theory particles generated by IAF insertion and removal should be merged together to evaluate the procedure-related risk of intimal manipulation. However, this was not done because of obvious statistical results from the individual IAF maneuvers.

Similar to IAF, the insertion of the aortic cannula produced embolic particles with different morphological appearances. Our finding supports the observations by Barbut and Caplan ${ }^{8}$ on the basis of transcranial Doppler recordings during surgical intervention. Aortic crossclamp removal also generates a significant output of embolic material, ${ }^{8}$ which our study again confirms. Repeated ACC within the same aortic location caused the particle load to decrease, which agrees with our previous findings. ${ }^{12}$ The findings have clinical relevance for multiple aortic crossclamps during surgical intervention by using a fibrillating heart technique, as well as repeated partial clamping for proximal anastomoses.

The spectrum of macroscopic calcified debris is of greatest relevance for CVA. The clinical importance of semitransparent debris is more speculative because this form of debris is assumed to be shed intimal cells. These particles are more numerous than their calcified macroscopic counterparts.
The maximum diameter of a macroscopic particle produced by the IAF was approximately $3 \mathrm{~mm}$ and $9 \mathrm{~mm}$ for calcified and cellular debris, respectively. The corresponding size for ACC-generated particles was somewhat larger. The microscope revealed detail of the structure of the debris, of which cellular particles exceeded calcified particles by a multiple of 10. For both types of microscopic spectra, the recorded maximum diameter was in the range of $0.5 \mathrm{~mm}$, a finding that might be more the result of the microscopic viewing size than the actual size of the particles. The above diameter values can be compared with the pore size of the IAF filter mesh, which was $0.12 \mathrm{~mm}$. In anatomic terms the arterial diameter in the circle of Willis is approximately $5 \mathrm{~mm} .{ }^{17}$ The minimum capillary diameter in the microcirculation is about $5 \mu \mathrm{m} .{ }^{18}$

Our experimental model can be criticized for the use of cadaveric material because postmortem changes might have affected the results. However, the pathologic status of blood vessels is known to be durable in the early postmortem period, ${ }^{19,20}$ and furthermore, calcified material is inorganic. All tested subjects had various degrees of atherosclerotic disease and had many common clinical traits with patients undergoing routine cardiac surgery, although the cohort was not perfectly matched. Our study used a simplified retrograde perfusion model, which was necessitated for technical reasons. The surgical methodology in the study was simple, and was nearly identical with that performed in the operating room. Because of time-consuming experimentation and few available cases, the statistical power is limited. However, the model showed good reproducibility.

We conclude that IAFs collect particles. However, IAF manipulation generates embolic debris on its own, possibly from shed intimal particles that escape capture. This was observed at both IAF insertion and removal. Aortic cannulation and ACC were additional sources of embolic debris. The clinical importance of these findings remains to be shown.

We thank Mrs Anne-Marie Österdahl, Mr Dan Nylund, Dr Eva Lundin, and Dr Karin Sixtensdotter Graffmo, Department of Clinical Pathology, Umeå University Hospital.

\section{References}

1. Kronzon I, Tunick PA. Atheromatous disease of the thoracic aorta: pathologic and clinical implications. Ann Intern Med. 1997;126:629-37.

2. Währborg P, Booth JE, Clayton T, Nugara F, Pepper J, Weintraub WS, et al. Neuropsychological outcome after percutaneous coronary intervention or coronary artery bypass grafting: results from the Stent or Surgery (SoS) Trial. SoS Neuropsychology Substudy Investigators. Circulation. 2004;110:3411-7.

3. Okamura A, Ito H, Iwakura K, Kawano S, Inoue K, Maekawa Y, et al. Detection of embolic particles with the Doppler guide wire during coronary intervention in patients with acute myocardial infarction: efficacy of distal protection device. J Am Coll Cardiol. 2005;45:212-5.

4. Ahonen J, Salmenpera M. Brain injury after adult cardiac surgery. Acta Anaesthesiol Scand. 2004;48:4-19.

5. Boivie P, Edström C, Engström KG. Side differences in cerebrovascular accidents after cardiac surgery: a statistical analysis of neurologic 
symptoms and possible implications for anatomic mechanisms of aortic particle embolization. $J$ Thorac Cardiovasc Surg. 2005;129:591-8.

6. van der Linden J, Hadjinikolaou L, Bergman P, Lindblom D. Postoperative stroke in cardiac surgery is related to the location and extent of atherosclerotic disease in the ascending aorta. J Am Coll Cardiol. 2001;38:131-5.

7. Vaage J, Jensen U, Ericsson A. Neurologic injury in cardiac surgery: aortic atherosclerosis emerges as the single most important risk factor. Scand Cardiovasc J. 2000;34:550-7.

8. Barbut D, Caplan LR. Brain complications of cardiac surgery. Curr Probl Cardiol. 1997;22:449-80.

9. Bergman P, Hadjinikolaou L, van der Linden J. Aortic atheroma is related to number of particulates captured by intra-aortic filtration in CABG. Eur J Cardiothorac Surg. 2002;22:539-44.

10. Harringer W. Capture of particulate emboli during cardiac procedures in which aortic cross-clamp is used. International Council of Emboli Management Study Group. Ann Thorac Surg. 2000;70:1119-23.

11. Reichenspurner H, Navia JA, Berry G, Robbins RC, Barbut D, Gold JP, et al. Particulate emboli capture by an intra-aortic filter device during cardiac surgery. J Thorac Cardiovasc Surg. 2000;119:233-41.

12. Boivie P, Hansson M, Engström KG. Embolic material generated by multiple aortic crossclamping: a perfusion model with human cadaveric aorta. J Thorac Cardiovasc Surg. 2003;125:1451-60.
13. Caplan L. Saving the brain during coronary artery bypass grafting. Lancet Neurol. 2003;2:211.

14. Hedberg M, Boivie P, Edström C, Engström KG. Cardiovascular accidents after cardiac surgery: An analysis of CT scans in relation to clinical symptoms. Scand Cardiovasc J. 2005;39:299-305.

15. Schmitz C, Weinreich S, White J, Oengoeren I, Schneider R, Schneider $\mathrm{D}$, et al. Can particulate extraction from the ascending aorta reduce neurologic injury in cardiac surgery? J Thorac Cardiovasc Surg. 2003;126:1829-38.

16. Banbury MK, Kouchoukos NT, Allen KB, Slaughter MS, Weissman NJ, Berry GJ, et al. ICEM 2000 Investigators. Emboli capture using the Embol-X intraaortic filter in cardiac surgery: a multicentered randomized trial of 1,289 patients. Ann Thorac Surg. 2003;76:508-15.

17. Vogel FS, Fuller GN, Bouldin TW. Pathology. 3rd ed. New York: Lippincott-Raven; 1998. p. 1474.

18. Potter RF, Groom AC. Capillary diameter and geometry in cardiac and skeletal muscle studied by means of corrosion casts. Microvasc Res. 1983;25:68-84.

19. Sachs ES. Effects of autolysis in vitro on the fine structure of human aortic intimal cells. J Atheroscler Res. 1967;7:549-65.

20. Yla-Herttuala S, Nikkari T. Effect of post-mortem time on the biochemical composition of coronary arteries. Atherosclerosis. 1985;56: $1-10$.

Access to The Journal of Thoracic and Cardiovascular Surgery Online is reserved for print subscribers!

Full-text access to The Journal of Thoracic and Cardiovascular Surgery Online is available for all print subscribers. To activate your individual online subscription, please visit The Journal of Thoracic and Cardiovascular Surgery Online, point your browser to http://www.mosby.com/jtcvs, follow the prompts to activate your online access, and follow the instructions. To activate your account, you will need your subscriber account number, which you can find on your mailing label (note: the number of digits in your subscriber account number varies from 6 to 10). See the example below in which the subscriber account number has been circled:

\section{Sample mailing label}

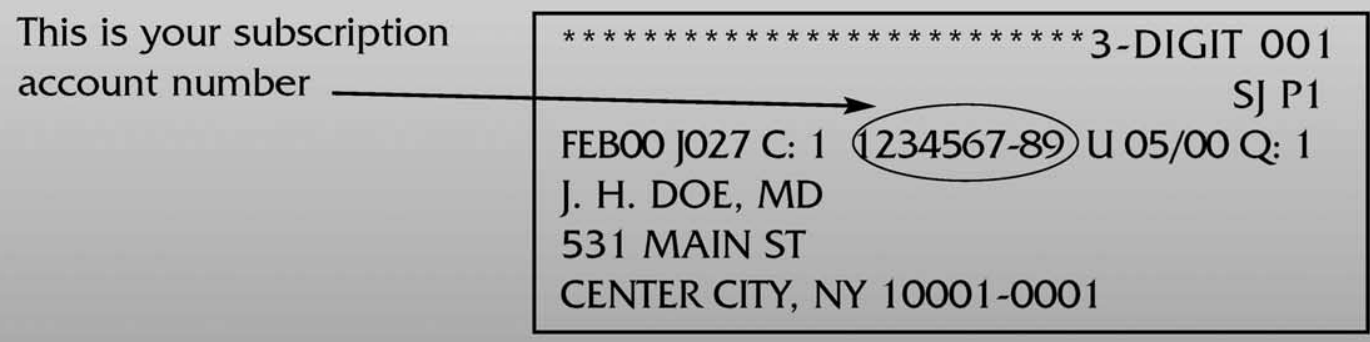

Personal subscriptions to The Journal of Thoracic and Cardiovascular Surgery Online are for individual use only and may not be transferred. Use of The Journal of Thoracic and Cardiovascular Surgery Online is subject to agreement to the terms and conditions as indicated online. 